\title{
ERMANNO STRADELLI IN AMAZZONIA: \\ L'ORO DELLA FORESTA E LA LEGGENDA DEL JURUPARY
}

\author{
ERMANNO STRADELLI NA AMAZÔNIA: \\ O OURO DA FLORESTA E A LEGGENDA DEL JURUPARY
}

\author{
Livia Raponi \\ Istituto Italiano di Cultura di San Paolo \\ livia.raponi@gmail.com
}

\section{Resumo}

Ermanno Stradelli (1852-1926), explorador italiano ainda desconhecido do grande público, deu uma imensa contribuição para a etnografia das populações amazônicas, em particular no âmbito linguístico e da etnografia religiosa. Pioneiro na coleta, documentação e divulgação de narrativas mitológicas indígenas, nos entregou um dos textos mais relevantes da literatura ameríndia, a Leggenda del Jurupary.

O presente ensaio visa examinar alguns aspectos e peculiaridades da Leggenda, que ainda hoje é referência para os antropólogos, por ser a versão mais completa e fiel do mito do herói-legislador dos povos do Alto Rio Negro.

Documento etnológico e obra literária ao mesmo tempo, é considerada por alguns estudiosos um texto sagrado; o seu tradutor, Ermanno Stradelli, nos aparece como um refinado intérprete e mediador da voz e do imaginário dos povos da floresta.

Palavras-chave: exploradores, Amazônia, etnografia indígena, mitos sul-americanos, literatura ameríndia, mediação linguística e cultural. 


\begin{abstract}
Ermanno Stradelli, an Italian explorer still unknown to the public, gave a very consistent contribution to the ethnography of Amazonian peoples, especially in the field of linguistics and ethnography of religion. A pioneer in collecting, preserving and sharing the mythical narrative of the natives, he is responsible for one of the major texts of Amerindian literature, the Leggenda del Jurupary.

This paper aims to study some aspects and peculiarities of the Leggenda, one of the most accurate version of the myth of the lawmaker hero of the tribes of the Upper Rio Negro and still today a reference to the anthropologists. An ethnological document and literary work at the same time, it is considered by some scholars as a sacred text; its translator, Ermanno Stradelli, appears as a distinguished interpreter and a mediator of the voice and the imaginary of the peoples of the forest.
\end{abstract}

Key-words: Explorers, Amazonia, Indigenous Ethnography, South American myths, Amerindian Literature, Linguistic and Cultural Mediation.

\title{
Sintesi
}

Ermanno Stradelli (1852-1926), esploratore italiano ancora sconosciuto al grande pubblico, diede un immenso contributo all'etnografia delle popolazioni amazzoniche, soprattutto in ambito linguistico e dell'etnografia religiosa. Pioniere nella raccolta, documentazione e divulgazione della narrativa mitologica indigena, ci ha lasciato uno dei principali testi della letteratura amerindia, la Leggenda del Jurupary.

In quest'articolo ci si propone di esaminare alcuni aspetti e peculiarità della Leggenda, che ancora oggi, per il fatto di essere la versione più completa e fedele del mito dell'eroe legislatore delle tibù dell'Alto Rio Negro, è opera di riferimento per gli antropologi. Documento etnologico e testo sacro ad un tempo, è ritenuta da alcuni studiosi un testo sacro; il suo traduttore, Ermanno Stradelli, ci appare come raffinato interprete e mediatore della voce e dell'immaginario dei popoli della foresta.

Parole-chiave: esploratori, Amazzonia, etnografia indigena, miti sudamericani, letteratura amerindia, mediazione linguistica e culturale. 
«Peregrino, d'extranhas terras vindo, mas desta terra, mais que filho, amante, a fraca voz elevo, a quem dá força o amor e canto (...)» ${ }^{1}$

Ermanno Stradelli, Ajuricaba

\section{Introduzione}

Ermanno Stradelli (Borgotaro, Piacenza 1852 - Manaus, 1926), esploratore, etnografo, linguista e scrittore, è inscindibilmente legato all'Amazzonia brasiliana. Giunto a Manaus nel 1879, il giovane conte di Piacenza inizierà un percorso di viaggi ed immersioni nell'ambiente naturale ed umano, che lo porterà in pochi anni a divenire uno dei maggiori conoscitori della regione, tanto sotto il profilo idrografico e morfologico, quanto sotto quello antropologico e culturale.

Navigatore instancabile dei principali affluenti del rio-mar, tra i primi a rilevare l'intero corso del fiume Uaupés, che percorse per più di 700 chilometri, autore di carte geografiche accurate ed osservatore attento del paesaggio, Stradelli raggiunse risultati altissimi nello studio delle popolazioni, del loro rapporto col territorio e delle loro manifestazioni culturali.

Nel suo avvicinarsi alle comunità indigene, fece uso di un metodo e di una sensibilità marcatamente antropologici: molto prima che tale strumento fosse consapevolmente teorizzato, realizzò una vera e propria «ricerca sul campo», basata sulla convivenza presso le società oggetto di studio e sulla conoscenza approfondita della lingua dei nativi. Convinto che ciascun elemento di una cultura andasse interpretato all'interno del contesto di quest'ultima, adottò un approccio al lavoro etnografico che può ben definirsi «osservazione partecipante» (MALINOWSKI, 1922, pp. 6-25), riuscendo a far proprio il punto di vista dell'indio, a comprenderne il modo 
di pensare e di vedere la vita, a condividerne le difficoltà e le sfide che ne minacciavano la sopravvivenza.

Del suo sguardo carico d'empatia, sereno e sensibile, abbiamo traccia nei numerosi ritratti che realizzò nel corso dei suoi viaggi, da brillante pioniere della fotografia nella foresta amazzonica ${ }^{2}$.

Rispettato ed amato dagli indigeni, che, proprio per la capacità, grazie alle tecniche dello sviluppo fotografico, di «far apparire le persone», lo consideravano un pajé (sciamano), come pure per i poteri straordinari che il microscopio, che portava con sé, gli conferiva, potè avere accesso alle zone interdette ai bianchi, partecipare ai rituali sacri dai quali questi venivano esclusi, raccogliere ampie testimonianze di una sapienza antica e segreta, tramandata oralmente di padre in figlio. Era soprannominato, per mettere in risalto quelli che gli indios consideravano attributi soprannaturali, тауиа raira e cioè «figlio del grande serpente» ${ }^{3}$.

A ciò deve aggiungersi la forte inclinazione per le lettere, manifestata già da giovane in alcune prove poetiche ${ }^{4}$ e stimolata ed arricchita dal contatto con l'affascinante patrimonio della tradizione orale degli indios del fiume Uaupés, al quale si dedicò con passione, impegnandosi a fondo nella raccolta e divulgazione di svariate leggende mitologiche. Completa il quadro la lucidità nell'analisi delle strutture socioeconomiche, che ne fece un attento osservatore dell'organizzazione sociale e della vita materiale dei popoli della foresta ed un precursore nell'impostare correttamente i termini della questione indigena.

Nel panorama degli esploratori italiani dell'epoca, la sua figura risalta per diversi tratti: la scelta del Brasile piuttosto che dell'Africa, l'ampiezza degli interessi, tanto geografici quanto etnologici ed umanistici, l'approccio rispettoso, paritario e privo di pregiudizi etnocentrici nei confronti delle civiltà indigene, la capacità di rapportarsi ed entrare in simbiosi con l'altro e il diverso.

A colpire è anche la linearità del percorso esistenziale, segnato in modo decisivo dall'incontro con le popolazioni che vivevano lungo i fiumi Uaupés e Rio Negro. Per lo studioso, l'Amazzonia non sarà semplicemente la meta di un viaggio, né la terra straniera in cui si perseguono la ricchezza e il successo - come accadde per molti europei emigrati in quegli anni in Brasile - bensì la patria necessaria, alla quale si è 
legati da una passione e un desiderio, in gran parte irrazionali, che assoggettano e non lasciano scampo.

Primogenito di un'antica famiglia della nobiltà piacentina, il giovane Ermanno seppe trasformare l'attrazione per i viaggi in paesi lontani ed esotici, alimentata dalla lettura dei romanzi d'avventura allora di moda, in un vero e proprio progetto di vita.

Studente di giurisprudenza all'università di Pisa, decise, alla morte del padre, di diventare esploratore, geografo ed etnologo. Abbandonati i manuali di diritto, cominciò a studiare, da autodidatta, le discipline che riteneva dovessero essergli utili: lo spagnolo e il portoghese, topografia e disegno, astronomia e meteorologia, ornitologia ed entomologia, botanica, farmacia ed omeopatia, etnologia. S'impadronì inoltre perfettamente delle tecniche della fotografia.

Partito per l'America Latina nel 1878, a 27 anni, con il patrocinio morale della Società Geografica Italiana, della quale era socio, e tra le forti perplessità della famiglia, Stradelli, nelle sue spedizioni, potè contare esclusivamente sul proprio entusiasmo e sulle proprie risorse.

Dopo aver trascorso alcuni mesi a Manaus, presso i missionari francescani, per perfezionare il portoghese, viaggiò per il Rio Purus e i suo affluenti (1880); successivamente, risalì il Rio delle Amazzoni sino a Fonte Boa e Loreto, dove entrò in contatto con la lingua nheengatu ${ }^{5}$ e se ne appassionò. Lo studio e la raccolta di termini e locuzioni di questo idioma melodioso lo accompagnerà in tutte le future esplorazioni, sino a prendere corpo, più tardi, in un'opera di grandissimo valore, i Vocabolari nheengatu-portoghese e portoghese-nheengatu (1929).

Sul Rio Juruá, ebbe occasione di assistere all'estrazione del caucciù e si fermò qualche tempo per studiare tutte le fasi del lavoro dei seringueiros ${ }^{6}$. Colpito dalla malaria, fu costretto a tornare a Manaus per la convalescenza, ma ripartì quasi subito per il fiume Uaupés (1881), lungo il cui corso compivano le loro ricerche gli studiosi João Barbosa Rodrigues, Antonio Brandão de Amorim, e l'indio Maximiano José Roberto, con i quali condivise l'interesse per le popolazioni rivierasche e strinse amicizia ${ }^{7}$. Incuriosito dalla cultura degli indios Tariana, prese a studiarne la lingua - di famiglia aruaco - e la tradizione orale. Raccolse, inoltre, svariati oggetti d'interesse etnografico, tra cui armi, amuleti ed ornamenti rituali, che inviò in Italia e che sarebbero stati esposti alla Mostra Colombiana di Genova del 1892.

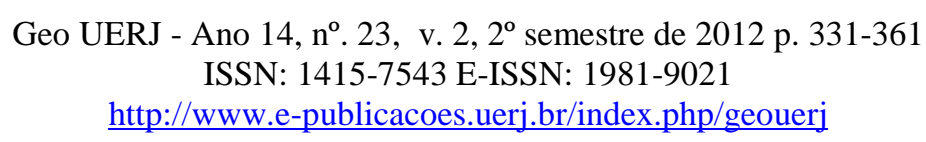


Nel 1882, accompagnò, come attaché en amateur, la Commissione Brasiliana per la delimitazione delle frontiere con il Venezuela, navigando lungo i fiumi Padauiri e Maiari, Rio Branco e Rio Negro. Lo stesso anno ripercorse il Uaupés sino a Jauaretécachoeira e navigò lungo il Madeira. Fece parte, nel 1884, della spedizione per la pacificazione degli indios Crichanás del fiume Jauaperi, guidata da Barbosa Rodrigues: questo viaggio fu molto significativo perché gli permise di vedere con i propri occhi i risultati nefasti dei metodi utilizzati dai bianchi, che da secoli, con l'uso della violenza e della sopraffazione, avevano generato negli indigeni un odio ed una ribellione difficili da estirpare.

Nel 1887, assieme all'amico Augusto Serra, concepì l'idea di una vera e propria spedizione da geografo, con l'obiettivo di scoprire le sorgenti del fiume Orinoco. Quest'impresa, che avveniva sotto l'egida istituzionale della Società Geografica, e lo vide accolto con ogni onore dal Presidente della Repubblica del Venezuela, non ebbe, per la defezione di Serra e il venir meno dell'equipaggiamento che questo avrebbe dovuto portare con sé dall'Italia, risultati significativi. Seguirà un viaggio lungo il Rio Branco, nel 1888, e, nel 1890-91, un'ultima spedizione lungo l'amato Uaupés ${ }^{8}$.

Rientrato in Italia solo tre volte, la prima per concludere gli studi di diritto e far pratica d'avvocatura (1884-1887), e successivamente per due brevi soggiorni, nel 1897 e nel 1901, Stradelli trascorse la maggior parte dell'esistenza in Amazzonia. Naturalizzatosi brasiliano nel 1893, entrò nella magistratura e servì come pubblico ministero per più di vent'anni, a Manaus, Labrea e Tefé. La sua vita si svolse operosamente e semplicemente, divisa tra la professione e il paziente lavoro di sistemazione delle voci dei Vocabolari, sino a quando, ammalatosi di hansenismo, fu esonerato dai pubblici uffici. Terminò in povertà e solitudine la sua esistenza, nel lebbrosario di Umirizal, a Manaus.

Furono proprio i Vocabolari, ai quali dedicò lunghi anni e infinite energie, senza avere la soddisfazione di vederli pubblicati ${ }^{9}$, ad attrarre l'interesse di Luís da Câmara Cascudo: ben oltre un semplice dizionario, essi costituiscono un' autentica enciclopedia della civiltà amazzonica e, come sottolinea Gordon Brotherston (2004, p. 345), sono una fonte ancora attuale e ricchissima d'informazione sulla complessità della vita nella foresta. 
In occasione del decennale della morte, l'etnologo brasiliano volle ricordare la figura dell'esploratore attraverso la monografia En Memória de Stradelli (1936), che racchiude un paziente lavoro di ricostruzione della biografia.

Ł̀ a questo illustre pesquisador che dobbiamo il riscatto dall'oblio di Ermanno Stradelli, che nonostante il valore e l'ampiezza delle opere realizzate nell' arco di più di quarant'anni, non ottenne riconoscimenti né soddisfazioni dai suoi contemporanei. Sorprende d'altra parte come tuttora il personaggio sia noto solo alla ristretta cerchia degli specialisti, ed il fatto che non abbia destato, tra gli studiosi italiani, l'interesse che meriterebbe ${ }^{10}$. Una migliore conoscenza e valorizzazione del validissimo apporto di Stradelli all'etnografia, alla linguistica ed alla letteratura amerindia, si riscontrano presso gli antropologi brasiliani e latino-americani ${ }^{11}$.

In tale quadro, è apparsa più che opportuna l'attenzione riservata, nell'ambito della manifestazione «Momento Italia-Brasile» promossa dal Ministero degli Affari Esteri italiano in Brasile, all'esploratore emiliano, al quale sono state dedicate, dall'Università Statale di Rio de Janeiro, un'esposizione ed una tavola rotonda ${ }^{12}$.

Le fotografie dello stesso Stradelli, che hanno costituito il nucleo principale della mostra, raccontano non solo il territorio ed i paesaggi attraversati, ma anche il contesto umano, lo stile di vita e i costumi delle popolazioni via via incontrate, ponendosi come perfetto contrappunto ai diari di viaggio, inviati, a partire dal 1887, all'allora Reale Società Geografica Italiana e pubblicati sulla rispettiva rivista.

Definito da Câmara Cascudo, nel testo citato, «esploratore nato», «etnografo spontaneo»e «amazzonense spirituale», Stradelli seppe far fronte, nonostante gli scarsi mezzi a disposizione e le risorse umane limitate, alle mille insidie della navigazione nel bacino fluviale amazzonico e della foresta vergine. Centrale, come si è detto, fu la regione dell'Uaupés, fiume mitico carico di favole e leggende, che percorse per tre volte da foce a monte, osservando e annotando scrupolosamente ogni cosa, i dati geografici e naturalistici, e, in misura maggiore, quelli antropologici e culturali.

È d'altra parte nella veste di studioso e divulgatore del fabulario degli indios Tariana e Tukano, espresso nella particolarissima tradizione orale, che ha raccolto i risultati più significativi. Accanto ai Vocabulários, l'opera più meritoria dello studioso è La leggenda del Jurupary, ampia e articolata versione dell'epopea mitica dell'eroe 
civilizzatore, apparsa nel 1890 sul «Bollettino della Società Geografica Italiana» (STRADELLI, 1890b) ${ }^{13}$.

\section{Sulle tracce del mito di Jurupari}

Per primo, Stradelli rese possibile la conoscenza di questo importantissimo mito amerindio nella sua formulazione originaria: con la Leggenda, raccolse e consolidò la tradizione indigena secondo la quale Jurupari è l'antenato legislatore al quale si devono le norme che reggono la convivenza e l'assetto sociale presente, il progenitore mitico semi-divino che, per la sua fondamentale opera civilizzatrice, è temuto, rispettato ed oggetto di culto. Rappresenta l'entità intermediaria tra dio e gli uomini comune a tutte le religioni: concepito da una vergine, è inviato dal Sole sulla Terra per ristabilire un equilibrio che per varie ragioni si è alterato, attraverso una profonda azione di riforma delle leggi e dei costumi adottati all'interno della comunità. Si tratta di un eroe culturale positivo, trasformatore, portatore di contenuti etici e morali.

Il testo giunse a coronamento di un'approfondita indagine dello studioso, volta a restituire alla figura di Jurupari, corrotta nei secoli da molteplici mistificazioni, la fisionomia originaria.

Al tempo dei primi viaggi di Stradelli lungo il fiume Uaupés (1881 e 1882), sulla sua rappresentazione gravava ancora il peso dell'interpretazione dei primi missionari, che gli studiosi avevano in gran parte condiviso. Agli occhi dei padri giunti nel sedicesimo secolo nella regione amazzonica, i popoli «selvaggi» che l'abitavano, privi della nozione del Dio del cristianesimo, vivevano in balia di mille superstizioni e false credenze, che li tenevano lontani dalla verità e dalla salvezza.

Cercata invano, nel pantheon amerindio, una divinità che potesse essere associata all'idea del dio cristiano, immateriale, eterno e onnipotente, si erano dovuti accontentare di identificarlo con «Tupã», termine nhengatu con il quale gli indios si riferivano al tuono, e che, pur declinato in tutti i sacri attributi nelle prediche e nelle orazioni, non giunse mai ad assumere una connotazione religiosa (CÂMARA CASCUDO, 1947, p. 80 e segg). 
Non avevano invece dovuto compiere molti sforzi per individuare un'entità che personificasse il maligno: il potente e rispettato Jurupari, che, d'aspetto mostruoso, si presentava ai nativi al suono lugubre di lunghi flauti, nel corso di feste che prevedevano misteriose danze e flagellazioni, sembrava l'incarnazione stessa del demonio cristiano.

L'identificazione di Jurupari con il diavolo ebbe più successo di quella di Tupã con l'onnipotente ${ }^{14}$. Se l'idea di un'entità creatrice immateriale restava per gli indigeni vaga e priva di consistenza, quella di diavolo trovava un terreno propizio nella convinzione, in particolare diffusa presso le tribù dell'interno, di vivere circondati da una pluralità di spiriti terribili e malefici che costituivano una costante minaccia. Istigati dai catechizzatori, gli indios iniziarono ad attribuire al «diavolo», le sofferenze e i turbamenti che queste entità gli causavano, e così chiamarono, tra gli altri, lo spirito di anhanga («spettro, fantasma e apparizione che reca tristi presagi», secondo il vocabolario di Stradelli), cercando, per difendersene, la protezione dei missionari ${ }^{15}$. Avvenne così che questi spiriti maligni, associati al diavolo cattolico, trasmisero a quest'ultimo, in un reciproco scambio di attributi, alcune loro caratteristiche; in virtù di questa commistione anche Jurupari, che con il diavolo veniva ad essere identificato, assumeva tratti propri dei demoni della foresta.

Un'ulteriore mistificazione doveva risultare dal contatto con lo spiritismo magico di provenienza negro-africana, che trasformò Jurupari nel «fantasma che di notte disturba il sonno e serve di spauracchio ai bambini», rovesciandone le prerogative originarie (PELOSO, 1984, p. 33).

Per tutto il XVII e XVIII secolo, il culto di Jurupari, condiviso da tutte le tribù del bacino amazzonico, era apparso ai missionari una vera «religione demoniaca nazionale», con tanto di sacerdoti ${ }^{16}$.

Quest'immagine alterata traeva in inganno, ancora sul finire dell'Ottocento, anche gli studiosi più attenti. Per José Vieira Couto de Magalhães, che si proponeva di affrontare lo studio della religione degli indios «come un naturalista» - ovvero basandosi sull'osservazione dei fatti -, Jurupari non è più il diavolo dei missionari, ma conserva gli attributi del fantasma notturno (1876, p. 83) ${ }^{17}$.

Altri studiosi, negli stessi anni in cui il generale de Magalhães attraversava le foreste dell'entroterra brasiliano, cominciavano a documentare il culto di Jurupari così come lo vedevano praticato dagli indigeni, con un approccio scientifico ed etnologico. Se già 
Alexander von Humboldt aveva riscontrato, tra gli indios dell'Alto Orinoco, danze rituali che prevedevano l'uso di strumenti musicali interdetti alle donne, fu il naturalista Alfred Russell Wallace il primo a registrare in modo oggettivo il culto di Jurupari e le cerimonie ad esso legate.

L'esploratore viaggiò per il Uaupés (1852) spingendosi sino a Jauareté, e lasciò osservazioni chiare e affidabili sulla mitologia e la pratica religiosa degli indios. Fornì la prima descrizione accurata degli strumenti che venivano utilizzati nelle feste rituali e delle melodie che ne venivano prodotte; documentò anche la proibizione alle donne di avvicinarsi ai sacri flauti o anche solo di vederli, e la terribile sanzi one che accompagnava la violazione di questo divieto.

Un pò prima che facesse notte, udimmo un suono, come di tromboni e di bassi, che veniva dal fiume in direzione del villaggio. Poco tempo dopo comparvero otto Indi, ognuno dei quali suonava uno stumento simile ad un fagotto. Portavano quattro paia di questi strumenti di differenti grandezze, con i quali facevano una musica selvaggia, tuttavia gradevole. Tutti suonavano i loro strumenti nello stesso tempo, formando nell'insieme un concerto tollerabile. [...] I detti strumenti sono fatti di corteccia di albero arrotolata in spirale ed hanno anche un boccale fatto di foglie. [...] Dal momento in cui comincia la musica, nessuna donna, vecchia o giovane, potrà rientrare [nella maloca], perché ciò fa parte delle strane superstizioni degli indi Uaupés. Si considera così pericoloso che una donna veda uno di quegli strumenti che, quando così accade, ella è punita con la morte [...]. (Cit. in BIOCCA 1965, pp. 215-216).

Il momento culminante del rituale era costituito, presso gli indios Tariana e Baniwa, dall'apparizione di Jurupari, inscenata da un danzatore che indossava la maschera sacra della divinità (makakaraua) ed altri ornamenti rituali.

È proprio questa maschera ad essere al centro, nel 1883, di una vicenda che coinvolse due frati francescani e gli indios della missione di Ipanoré (alto Uaupés), mostrando tutta l'importanza e la vitalità di questo culto, che continuava ad esistere, per gli indigeni, accanto alle messe e al catechismo ${ }^{18}$. Tale episodio, raccontatogli in seguito dal protagonista, padre Illuminato Coppi, avrà grande influenza sul successivo lavoro di Stradelli.

Entrato in possesso della maschera rituale, il missionario Coppi decise di dimostrare agli indios della missione, in modo irrevocabile, la falsità della credenza per la quale, alla vista della stessa, le donne sarebbero morte. Non era facile appropriarsi di tale Geo UERJ - Ano $14, \mathrm{n}^{\mathrm{o}} .23$, v. 2, $2^{\mathrm{o}}$ semestre de 2012 p. 331-361

ISSN: 1415-7543 E-ISSN: 1981-9021

http://www.e-publicacoes.uerj.br/index.php/geouerj 
oggetto, ma egli l'aveva estorto, insieme ad altri strumenti rituali, al pajé Ambrosio Picuita, che, tratto in salvo da una faida tribale, si trovava praticamente alla sua mercè ${ }^{19}$.

Questo infame simulacro è un tessuto rozzo di capello di donna e pelo di scimmia, maccaragua ha la forma di una grande sacchetta, che giunge sino alla cintura. Essa ha due grandi fori per porvi i bracci, due altri per vedervi e un terzo per respirarvi, terminando con un ciuffo di penne di vari colori, il Pagé che la indossa figura una paurosa e tetra maschera (COPPI, 1897, p. 43).

Quest'appropriazione e la successiva profanazione della maschera furono all'origine di una grande rivolta, che raccolse solidali tutti gli indios dell'Alto Rio Negro, costringendo per molto tempo all'abbandono delle missioni nell'intera regione. La descrizione che degli eventi fece padre Coppi, «con scarso rispetto della grammatica ma con appassionato fervore» (BIOCCA, 1964, p.7), documenta la totale incomprensione del culto di Jurupari:

Il simulacro diabolico era già nascosto dentro il pulpito, dove il Cagnoni vi ascese predicando, guardando io l'ordine in caso di rivolta già preveduta. A un tratto l'oratore prese in mano l'infame maschera, e mostrandola al popolo, un grido cupo e lugubre e pauroso uscì da quelle bocche impaurite e tremanti di terrore e spavento. Ne nasce una orribile confusione e colluttazione cercando tutti di uscire dal tempio, la cui porta tentai serrare, obbligando la gente a mirare il simulacro.

I pianti, i gridi, gli urli del popolo, e le minacce dei pagés furibondi assordavano, e tutti quali tigri addosso a noi per sbranarci. [...] La porta del tempio fu svelta dai suoi cardini, il tetto della chiesa spalancato, le finestre rotte, e noi due Missionari impegnati, [...] alla propria difesa, assaliti da quelle belve e leoni soffiando i pagés sopra il popolo sbigottivo e fuggitivo, per scacciare la cattiva influenza dei Missionari (!) [...] sentii la voce del compagno chiedendo soccorso [...] là volai intrepido nella lotta uscendone con le vesti lacerate e contusi, ma con il trionfo in mano, non avendo ceduto il diabolico simulacro (ibidem).

L'esploratore Henri Coudreau, incontrati i due missionari poco tempo dopo gli avvenimenti narrati, ne aveva raccolto una versione della leggenda di Jurupari che lo dipingeva come un demonio selvatico e terribile, e l'aveva pubblicata ${ }^{20}$.

Stradelli, che si stava documentando al riguardo, trovandosi in netto disaccordo con l'interpretazione accettata dal francese, ebbe il desiderio di andare più a fondo. 
Quando nel 1883 Coppi venne a Manaos dopo la presentazione della maschera del Jurupari e mi raccontò i segreti della 'religione del diavolo', come diceva, ultimamente svelati, questi mi parvero per la maggior parte esagerazioni del fantasioso, ma non altrettanto coraggioso francescano [...]. Vi si vedeva troppo quello stesso spirito prevenuto dei primi missionari, che tutto ciò che usciva dall'orbita cristiana, che aveva un aspetto nuovo, era per lo meno diabolico; e non vi prestai che un'attenzione molto relativa [...]. Volli averne il cuore netto e cominciai a riunire i miei pochissimi frammenti e cercare, interrogando qualche Uaupés, di raccapezzarmi come stava veramente la cosa (STRADELLI, 1890a, p. 453).

Il lavoro compiuto da Stradelli sul mito di Jurupari costituisce un fondamentale momento chiarificatore nell'intricata storia di false interpretazioni che lo avevano contraddistinto. Pur non essendo il primo studioso a soffermarsi sul tema, ebbe il merito fondamentale di liberare il campo dagli errori grossolani e dalle mistificazioni, riuscendo nella difficile impresa di recuperare il Jurupari autentico dall'oblio, e di restituirgli la posizione di primissimo piano che gli spetta nella cosmogonia amerindia.

Fin dal suo primo viaggio lungo il fiume Uaupés (1881), il giovane ricercatore era stato incuriosito dalla figura di Jurupari e aveva raccolto frammenti della leggenda dagli indigeni, ricavandone l'impressione che l'identificazione col diavolo era falsa, e che piuttosto ci si potesse servire, nell'interpretazione, del concetto greco di daimonion.

Nel Simposio Platone, per bocca della sacerdotessa Diotima, definisce il daimonion come una creatura intermedia tra il divino e il mortale, che agisce come interprete e messaggero tra gli dei e gli uomini (202 e). Il dio non si confonde con l'uomo, ma in virtù del demone, è possibile stabilire una comunicazione tra l'uno e l'altro: il demone trasmette agli dei le preghiere e i sacrifici degli uomini, e agli uomini i comandi o i favori da parte degli dei.

Questa intuizione iniziale lo accompagnò nel corso delle successive esplorazioni, nelle quali ebbe modo di osservare tribù delle più varie provenienze praticare il culto e rispettare le norme legate al Jurupari, che costituivano un vero e proprio sistema per il governo della comunità - una costituzione - e, ancora sul finire dell'Ottocento, influivano largamente sui costumi di popolazioni disseminate in un'area molto ampia, parallelamente a norme, abitudini e costumi introdotti ed imposti dai catechizzatori cristiani. 
Rafforzato nel suo proposito dalle esagerazioni di Padre Coppi, prese per buone da suoi autorevoli colleghi, Stradelli smascherò tutte le false accezioni del termine Jurupari, di cui darà in seguito conto nei Vocabolari : «il demonio o lo spirito maligno, secondo tutti i dizionari e i missionari [...]»(1929, p. 497). Rispetto alla persistente concezione di «fantasma notturno» - confortata dall'interpretazione della parola come «essere che viene al nostro giaciglio» - esprime una netta contrarietà, ritenendo che il nome «Iurupari» vorrebbe piuttosto significare «colui che sigillò la nostra bocca», da «iuru», bocca, e «pari», grata, reticolato di legno con cui gli indigeni chiudono le bocche dei fiumi per trattenere i pesci. Quest'interpretazione avrebbe per Stradelli il vantaggio di esprimere in modo efficace la parte fondamentale della missione di Jurupari, eroe riformatore, e cioè l'istituzione del segreto ${ }^{21}$.

Istituì delle feste, alle quali solo gli uomini possono prendere parte, e dei segreti, che solo questi ultimi possono conoscere. Le donne che assistono ai riti devono morire [...]. Ciononostante, non tutti gli uomini conoscono il segreto; solo gli iniziati, coloro che, giunti alla pubertà, dettero prova di saper sopportare il dolore, essere riservati e impavidi (ibidem).

È interessante sottolineare che, sempre nel Simposio, si allude a un'importantissima funzione del daimonion, legata al mantenimento della coesione della comunità e alla sfera dei riti magico-religiosi, che, come vedremo, caratterizza anche Jurupari:

« [...] poiché sta nel mezzo fra dei e uomini, colma lo spazio intermedio in modo che l'insieme resti saldamente connesso in tutte le sue parti. Nella sfera del demonico si svolge tutta la pratica divinatoria e l'arte dei sacerdoti in relazione ai sacrifici e alle iniziazioni e agli incantesimi e a ogni genere di profezia e di magia» (203a).

\section{La Leggenda di Ermanno Stradelli}

Elemento determinante, che coronò la ricerca dello studioso permettendogli di andare a fondo a proposito del Jurupari, fu l'incontro con Maximiano José Roberto, indio colto divenuto intimo amico e compagno di viaggi, che gli offrì una chiave d'accesso privilegiata al mondo mitico e religioso degli abitanti dell’Uaupés. 
La figura di Maximiano, guida e informatore di molti etnologi ed antropologi che viaggiarono nell'Alto Rio Negro sul finire del XIX secolo, ci giunge attraverso le brevi note di questi ultimi, che ne riconobbero il grande merito di aver raccolto e documentato svariate narrazioni tradizionali indigene ${ }^{22}$. Era, per parte paterna, della tribù dei Manaos, e per parte di madre, un Tariana; suo zio materno, Mandù, era il tuixáua (capovillaggio) di Jauareté ${ }^{23}$. Così lo ricorda Câmara Cascudo:

Capiva la lingua della foresta, dei fiumi e degli uccelli. Scopritore della vena luminosa, visse distribuendo l'oro del quale lui solo conosceva il tracciato invisibile. Ignorata e gigantesca intelligenza, semplice e generosa, forte e solitario nel suo sogno misterioso e affascinante (1936, p. 11).

Tra tutte le tribù dell'Uaupés, quella dei Tariana occupava una posizione di rilievo, possedendo una tradizione culturale, guerriera e politica superiore alle altre comunità del nord-ovest amazzonico, in virtù della quale era soprannominata «la tribù dei capi». Proprio dai Tariana, che si erano imposti nella regione combattendo e vincendo sulle altre popolazioni, pareva essersi irradiato il culto di Jurupari, con le norme ed i riti ad esso collegati.

All'amico italiano, per il quale nutriva profonda stima e rispetto, e non ad altri, Maximiano volle offrire «l'oro», e cioè un prezioso manoscritto in lingua nheengatu contenente un'estesa e articolata versione del mito ${ }^{24}$. Il testo era il frutto di una lunga e paziente opera di raccolta della tradizione orale di diverse tribù e, come spiega Stradelli, Maximiano era l'unico a poter realizzare l'impresa:

Egli cominciò raccogliendo la leggenda dall'uno e dall'altro, confrontando, ordinando le diverse narrazioni e sottoponendole alla critica dei diversi indigeni riuniti, cosicché oggi egli può assicurare di presentare la fedele espressione della leggenda indigena, di cui ha conservato quanto più ha potuto, perfino il colore della dizione; cosa del resto che non gli era difficile, non essendogli ignoti né il dialetto tucana, né il tariana, e conoscendo a fondo la língua geral, o nheengatu che si voglia chiamare; ed anzi spero che prima o poi pubblicherà come ha promesso il testo originale colla traduzione (STRADELLI, 1890a, p. 452).

Proprio grazie a questo manoscritto, Stradelli potè far luce su molti aspetti del mito e consegnarci un'epopea completa delle avventure dell'eroe legislatore. Se inizialmente 
aveva pensato di farne un riassunto, decise poi per una traduzione, il più possibile fedele all'originale ${ }^{25}$.

Va ricordata a questo proposito l'esperienza già maturata con la tragedia in versi Eiara: leggenda tupi-guarani, pubblicata in Italia nel 1885, nella quale aveva trasposto, in forma poetica, il tema mitologico dell'Eiara, signora delle acque, appreso direttamente dagli indios:

«la leggenda [...] è stata da me udita e raccolta sul posto nella mia non breve dimora nelle Amazzoni, cinque anni e mezzo circa, dalla bocca degli stessi indigeni, anzi non so se convenisse meglio chiamarla versione, come infatti è, se non dalla scrittura, dalla parola viva» (ivi, p.5) .

Apparsa nel 1890 sul «Bollettino della Società Geografica Italiana» ${ }^{26}$, la Leggenda del Jurupary, che comprende una pluralità di episodi ed è suddivisa in 27 capitoli, si apre con la storia di una fecondazione soprannaturale, che ha luogo «nel principio del mondo», in una tribù della Serra del Tenuí (Colombia) dove erano rimaste, a seguito di un'epidemia, solo le donne, alcuni anziani e un vecchio pajé.

Riunitesi a convegno presso il Lago Muypa, disobbedendo alla proibizione di quest'ultimo, le donne ne vennero tutte fecondate mentre facevano il bagno. Il pajé annunciò loro che la generazione futura avrebbe escluso per sempre il genere femminile dall'ingerenza negli affari importanti della comunità. Tra i nuovi nati vi era Seucy, che, rimasta incinta mangiando il frutto proibito del pihycan (pourouma cecropia efolia, un frutto ad acini) darà alla luce Jurupari.

Per il misterioso concepimento il giovane era destinato ad essere il signore dei Tenuiana, e a soli 15 anni fu acclamato capo del villaggio. Ricevuto dal padre, il Sole, il necessario per governare e riformare i costumi, oltre ad oggetti e amuleti che lo avrebbero aiutato nella sua missione, salì, a dorso di un'aquila reale, alla Serra della Luna, per avere da quest'ultima le vesti e le insegne del comando.

Il primo atto di Jurupari fu quello di presentarsi agli uomini, per insegnare loro l'agricoltura e rivelare le leggi che, a partire da quel momento, avrebbero dovuto regolare la loro condotta, da tenere rigorosamente segrete (in particolare, da non rivelare alle donne): 
Cominciò col dichiarare la sua costituzione duratura col nome di Jurupary fino a che il Sole illuminasse la Terra, proibendo assolutamente alle donne di prender parte alle feste degli uomini, quando stessero presenti gli strumenti speciali che dovevano essere distribuiti nella prossima adunanza inaugurale.

La violatrice di questa proibizione è perciò condannata a morte, condanna che dovrà essere eseguita da chiunque abbia pel primo conoscenza del delitto, sia questo pure padre, fratello, marito. Cosi l'uomo che mostrerà gli strumenti o svelerà le leggi segrete in vigore, ad una donna, sarà obbligato ad avvelenarsi, e quando non voglia, il primo che lo incontrerà dovrà dargli la morte $[\ldots]$.

Tutti i giovani che abbiano raggiunto l'età della pubertà devono conoscere le leggi di Jurupary, e prendere parte nelle feste degli uomini. [...] Tutti i suonatori di Jurupary devono avere alla mano una capeia (sferza, in banuia) per isferzarsi reciprocamente in ricordo del segreto che tutti devono conservare. Tutti quelli a cui toccherà qualche strumento del Jurupary (ciò accadrà alla futura luna piena) saranno obbligati d'andare ad insegnare per tutte le terre del Sole le cose ora dette [...] (STRADELLI, 1890b, p. 665).

A partire da questo atto solenne, che alcune donne spiano, venendo immediatamente castigate con la morte, Jurupari passa a consolidare le nuove norme e ad insegnare ai propri discepoli i riti sacri. Le donne tuttavia intendono privarlo del potere, e continuamente, con l'inganno, cercano di impadronirsi del segreto. Il primo a cadere nella trappola e a rivelare la legge di Jurupari è il vecchio Ualri, sedotto dalla giovane Diadue. Seguiranno altri tradimenti, ma sempre Jurupari interverrà per punire i trasgressori e ristabilire la sua costituzione.

Dalle ceneri di Ualri nasce un'altissima palma paxiúba (Socratea exorrhiza) dal cui tronco Jurupari ricava gli strumenti sacri («i sacri flauti»), da utilizzare nei rituali d'iniziazione e nelle feste. Questi assumeranno un ruolo fondamentale e simbolico nello svolgersi della vicenda e, poiché il loro possesso assicura il potere, saranno continuamente disputati tra gli uomini e le donne.

Momento centrale della narrazione è quello in cui Jurupari decide di rivelare ai suoi discepoli, riuniti nella capanna sacra, l'origine divina della sua gente. Racconta di come la giovane Dinari, fuggita dal suo villaggio e rifugiatasi nel bosco, avesse sposato il capo della tribù degli Agami (passeri) e come dalla loro unione fossero nati due gemelli, Pinon e Meenspuin, che avevano forma umana e il corpo coperto di stelle. Pinon è il 
progenitore mitico della specie, il pajé che, nell'episodio descritto all'inizio della storia, darà vita alla nuova generazione.

Solo alla fine della Leggenda, terminata l'opera di diffusione della nuova legge, Jurupari svelerà al suo fedele discepolo Caryda la sua missione segreta, che è una «missione impossibile»:

«Oggi, o Caryda, è l'ultimo giorno che noi restiamo insieme e prima di separarci voglio contarti il segreto della mia missione sopra la terra. Il Sole dacché nacque la Terra, cercò una donna perfetta, per chiamarla vicino a sé, ma siccome fino ad oggi non la ha incontrata, mi affidò parte del suo potere per vedere se in questo mondo potrà esservi una donna perfetta».

«E qual è la perfezione che il Sole desidera?»

«Che sia paziente, che sappia guardare un segreto e che non sia curiosa. Nessuna donna oggi esistente sulla terra riunisce queste qualità: se una è paziente, non sa conservar segreto; se sa conservar segreto, non è paziente; e tutte sono curiose [...].

Quando la notte di oggi sarà a mezzo dobbiamo dividerci; io andrò ad oriente e tu seguendo il cammino del Sole andrai a ponente. Se un giorno il Sole, tu ed io ci incontreremo nel medesimo luogo, vorrà dire che sarà apparsa sulla terra la prima donna perfetta» (ivi, p. 835).

Il tema principale del racconto è quello dell'affermazione, ad opera dell'eroe riformatore, della nuova costituzione sui precedenti costumi, tema che viene ad intersecarsi e a sovrapporsi con quello della lotta tra gli uomini e le donne per il governo della comunità: «quando apparve, erano le donne che comandavano e gli uomini obbedivano, il che era contrario alle leggi del sole. Egli tolse il potere dalle mani delle donne e lo restituì agli uomini [...]» (STRADELLI, 1929, p.497)

Se, al principio della storia, la descrizione di una tribù composta da sole donne fa pensare ad una società matrilineare ed endogamica, giunta ad una fase di decadenza, le norme introdotte da Jurupari, che sanciscono la monogamia e il dominio degli uomini, possono essere viste in funzione del passaggio ad un sistema patriarcale ed esogamico, biologicamente più vitale e suscettibile d'espansione. La donna, proveniente da un'altra tribù e dunque straniera, viene a trovarsi in una posizione d'inferiorità ed isolamento, ed è più facilmente esclusa dalle questioni importanti della vita collettiva. Questa è, per Stradelli, la verità storica racchiusa nel mito di Jurupari.

L'asse dinamico della Leggenda è rappresentato dalle dislocazioni compiute dal protagonista, grazie all'utilizzo di un amuleto, per realizzare i propri obiettivi. L'azione

Geo UERJ - Ano 14, nº. 23, v. 2, $2^{\circ}$ semestre de 2012 p. 331-361

ISSN: 1415-7543 E-ISSN: 1981-9021

http://www.e-publicacoes.uerj.br/index.php/geouerj 
si svolge in tre luoghi diversi, che rappresentano altrettanti nuclei dello svolgimento narrativo: la Serra del Tenuí, nell'Alto Uaupés colombiano, luogo dell'origine storicomitica dei Tariana, dove all'inizio del racconto Jurupari rivela le sue leggi; i margini del fiume Aiari, affluente dell' Içana (alto Amazonas), dove fa costruire la sua capanna e dove vengono fabbricati, dalla palma nata dalle ceneri di Ualri, i primi strumenti sacri; la cascata di Jurupari (Jurupari-Cachoeira), nei pressi dell'attuale Mitú, dove hanno luogo le prime feste rituali e che sarà il centro d'irradiazione delle nuove leggi.

È interessante soffermarsi su questa geografia del mito, che ripercorre le diverse fasi dell'epopea dell'eroe: nato e cresciuto in Colombia, Jurupari s'insedia in Brasile, dove erige la sua dimora e dove avviene l'iniziazione dei discepoli; ritorna poi nella terra d'origine, per celebrare i primi riti, e da lì ne inizia la diffusione in un'area più ampia, a nord, lungo l'Orinoco (Venezuela), e a sud (Brasile).

Si può pertanto affermare che ci troviamo di fronte ad un mito tanto colombiano quanto brasiliano, che, per il fatto di interessare un ampio territorio e più paesi, può essere considerato autenticamente latino-americano, come suggerisce Sergio Medeiros (2002, p. 9).

La Leggenda presenta diversi elementi che l'avvicinano ad altre narrazioni indigene americane. Le dinamiche principali della storia, che ricorrono più volte nello snodarsi dell'azione, si prestano ad essere analizzate utilizzando le unità strutturali individuate da Alan Dundes (1963) per i racconti aborigeni nordamericani. In particolare, si ritrovano con frequenza nel testo tanto l'unità «squilibrio - riequilibrio», per la quale ad una rottura dell'equilibrio segue il ritorno alla normalità, quanto l'unità «proibizione violazione - conseguenza», descritte dallo studioso (cfr. ORJUELA, 1983, p. 142 e segg).

Non potendosene affrontare, in questa sede, un'analisi dettagliata, ci si limiterà ad alcune considerazioni sul valore e le peculiarità della formulazione di Stradelli.

Questa fu avvolta, sin dal principio, da una polemica circa l'autenticità del testo, resa possibile dalla circostanza che il manoscritto in nheengatu non fosse stato rinvenuto: immediatamente dopo la pubblicazione João Barbosa Rodrigues aveva affermato che non si trattava della trascrizione di una narrativa orale autentica, ma del frutto dell'estro letterario del conte, che pure aveva sostenuto di essersi limitato a tradurre il manoscritto «il più semplicemente possibile».

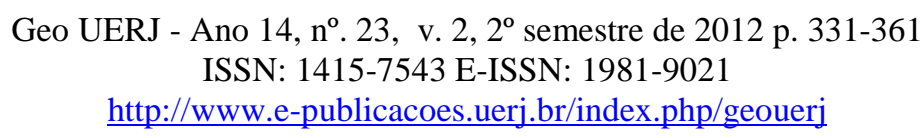


Si deve tuttavia tenere in considerazione il fatto che anche il brasiliano ambiva al manoscritto di Maximiano, e che glielo aveva più volte richiesto, senza ottenerlo. Affermando di essere stato il primo a conoscere la versione del mito trascritta dall'indio, Barbosa Rodrigues ci dà conferma della sua esistenza. Inoltre, la leggenda che pubblicò quasi in contemporanea $^{27}$, appare chiaramente come un riassunto della stesura di Stradelli, riconfermandone l'autenticità.

Altri, come Câmara Cascudo, ritennero che quest'ultimo non si fosse limitato a tradurre, ma, da vero e proprio co-autore del testo, avesse operato interventi strutturali per ottenere quell'unità narrativa di cui questo sarebbe stato privo, non compromettendo tuttavia, con quest'opera di sistemazione letteraria, la validità della Leggenda come documento etnologico (1926, p. 55 e segg .).

Appare tuttavia ragionevole ritenere, secondo ciò che lo stesso Stradelli illustra nel passo citato, che Maximiano, indio colto e dedito alla raccolta e al recupero della tradizione orale indigena, non si fosse limitato a trascrivere in língua geral una serie di leggende appartenenti al ciclo di Jurupari, ma avesse dato loro anche un certo ordine, unità e coerenza, fino a tracciare una vera e propria epopea dell'eroe mitico (cosi Lúcia Sá, 2002, p. 350-51).

Va inoltre evidenziato il fatto, giustamente segnalato da Sergio Medeiros (cit., p. 264), che questo materiale fu raccolto inizialmente non in lingua nheengatu, ma in lingua tariana. Il testo manoscritto che l'indio consegnò a Stradelli già era, pertanto, una traduzione dalla lingua parlata dagli indigeni dell'Uaupés alla lingua boa.

Il nostro studioso, alle prese con la traduzione in italiano, si sarebbe dunque già trovato di fronte ad un insieme narrativo strutturato e coeso, rispetto al quale lo sforzo sarebbe stato semmai quello di conservare il sapore originale - si veda la notevole ricchezza lessicale del testo, dovuta all'uso di vocaboli appartenenti a diverse lingue indigene - e l'andamento corale tipico del racconto orale.

Questa genesi complessa fa sì che, alla luce delle moderne acquisizioni della narratologia, la Leggenda si possa considerare di triplice autoria : la tradizione orale, dunque gli indios Tariana dell'Uaupés, collettivamente; il «compilatore», Maximiano; il «traduttore», Stradelli.

Il fatto che Stradelli ne sia indubbiamente co-autore, e sia potuto intervenire in qualche misura nella sua forma finale, non toglie al testo in italiano il carattere di Geo UERJ - Ano 14, nº. 23, v. 2, $2^{\circ}$ semestre de 2012 p. 331-361 ISSN: 1415-7543 E-ISSN: 1981-9021

http://www.e-publicacoes.uerj.br/index.php/geouerj 
traduzione da un documento autentico. Del racconto orale restano intatte le caratteristiche fondamentali: il ritmo piano della narrazione, la perfetta coincidenza tra la trama e la struttura del testo, l'assenza di qualsiasi commento, gli estesi dialoghi tra i personaggi, la grande varietà lessicale ${ }^{28}$.

La formazione letteraria dello studioso certamente influì sugli occasionali sprazzi lirici rintracciabili qua e là, in qualche abbellimento di gusto romantico, e in generale, nell'utilizzazione di un linguaggio che si distanzia da quello, asciutto ed essenziale, utilizzato abitualmente da antropologi ed etnologi. La presenza del traduttore è inoltre dichiarata nelle brevissime spiegazioni di termini in nheengatu inserite tra parentesi. Rispetto ad altre stesure, questa versione del mito si differenzia altresì per i frequenti sermoni di carattere morale pronunciati dal protagonista - elemento che contribuisce alla sua valenza letteraria - e per il fatto di racchiudere, al suo interno, un «mito dentro il mito» (quello di Pinon), in una prospettiva multitemporale che conferisce profondità e spessore al racconto.

Se è vero poi che la storia narrata è molto articolata e complessa e che vengono presentati numerosi episodi in uno svolgimento abbastanza rapido, questi tuttavia si susseguono in maniera logica, essendo ognuno la conseguenza degli accadimenti che lo precedono. Questa caratteristica strutturale, segnalata da Claude Lévi-Strauss in relazione ai miti dell'Uaupés (1978, [2010, p. 253]), può solo in parte attribuirsi, nel caso della Leggenda, al fatto che il materiale raccolto dagli indigeni fosse stato organizzato in sequenze dal compilatore, Maximiano José Roberto.

Secondo lo studioso francese, il ciclo di Jurupari sembrerebbe appartenere ad un genere mitologico a sé stante, di livello erudito ed esoterico, che lo avvicina alle narrazioni raccolte successivamente da Kurt Nimuendaju e Léon Cadogan tra i Guarani $^{29}$. Tale carattere potrebbe essere posto in relazione all'elevato livello di civiltà raggiunto, anticamente, dalle comunità amazzoniche, di cui vi è traccia in svariate testimonianze dei primi cronisti e di cui è indizio una tradizione orale che, per l'estrema complessità, la struttura articolata ed il tono mistico, può attribuirsi al contributo di scuole di saggi e filosofi (1973, [2004, p. 253] ).

Altri studiosi hanno di recente confermato quest'interpretazione, ritenendo la Leggenda di Stradelli un racconto cosmogonico di carattere sacro: in tal senso va interpretata la presenza, al suo interno, del mito di fondazione che fa luce sulle origini 
della specie. Come fa notare Lucia Sá, anche in Ayvu-Rapyta e Lendas da criação e destruição do mundo si dà conto di fasi della creazione anteriori all'azione degli eroi trasformatori, sconosciute ai non-iniziati. La storia dei gemelli ancestrali, cronologicamente anteriore alla nascita di Jurupari e da questo «rivelata» ai discepoli, indicherebbe che ci troviamo di fronte ad un testo sacro, probabilmente ottenuto grazie alla fiducia che Maximiano, discendente dalla tribù, possedeva tra i Tariana (2002, p. $351)$.

Ed è lo stesso antropologo francese ad offrirci illuminanti considerazioni sul tema della traduzione mitologica. A proposito della struttura del mito, che fa parte del linguaggio, sottolinea come sia caratterizzata da un elemento di originalità rispetto a tutti gli altri fatti linguistici: il mito può infatti essere definito come una modalità di discorso per la quale l'espressione «traduttore, traditore» perde ogni validità. La sua posizione, nella scala dei diversi modi di espressione linguistica, è opposta a quella della poesia, nonostante si sia tentato d'avvicinarli : la poesia è una forma di linguaggio estremamente difficile da tradurre in un'altra lingua, mentre il valore del mito, per misera che sia la traduzione, rimane.

Per quanto possiamo ignorare la lingua e la cultura della popolazione nella quale fu raccolto, un mito è percepito come tale da qualunque lettore, nel mondo intero. La sua sostanza non si incontra né nello stile, né nel modo di narrazione, né nella sintassi, ma nella storia che in esso è narrata. Il mito è un linguaggio, ma un linguaggio che lavora ad un livello molto elevato, nel quale il senso riesce, per così dire, a scollarsi dal fondamento linguistico nel quale inizialmente fu espresso (LÉVI-STRAUSS, 1958, [2008, p. 299] ).

Sempre sul tema della traduzione, va aggiunto che, secondo i più recenti approcci di coloro i quali si dedicano alla raccolta della narrativa indigena, dopo una prima fase di registrazione/trascrizione il più possibile fedele del racconto orale, è la traduzione creativa il processo che meglio permette di rendere, transponendoli in un diverso codice espressivo, gli elementi tipici di quella narrazione - ritmo, timbro di voce, intonazione, pause, gestualità - , che concorrono alla costruzione del significato (cfr. MINDLIN, 2007, p.15). Tali elementi performativi vanno persi tanto nella forma schematica del riassunto, quanto in una pretesa traduzione letterale, parola per parola. 
Appare pertanto definitivamente smentita e superata la concezione secondo la quale il passaggio attraverso la traduzione e la letterarietà di un testo sarebbero elementi che ne annullano il valore di documento etnologico.

In virtù delle considerazioni che precedono, la valenza letteraria del racconto di Stradelli, basato fedelmente su un documento che, per il fatto di provenire da un nativo, offriva le migliori garanzie d'attendibilità, appare semmai un elemento che ne innalza l'interesse ed il pregio.

Narrazione avvincente e testimonianza autentica ad un tempo, La Leggenda ci consegna, intatta, l'epopea del Jurupari, uno dei maggiori miti pre-colombiani, e va annoverata tra le prime, importantissime manifestazioni della letteratura amerindia. Per queste sue caratteristiche, costituisce ancora oggi una fonte imprescindibile per gli studiosi.

\section{Considerazioni conclusive}

Esaminando l'approccio di Stradelli alle civiltà indigene, espresso nelle diverse attività che portò avanti durante la sua vita in Amazzonia, si può individuare, come elemento unificante e distintivo, un certo sguardo, che, come sull'obiettivo della macchina fotografica, si posa dolcemente, con precisione e interesse, sull'oggetto incontrato.

Uno sguardo partecipe e attento, straordinariamente moderno perché sostenuto dalla consapevolezza dell'importanza e del valore del patrimonio culturale dei popoli della foresta, accompagnata dal senso dell'urgenza di doversi adoperare affinché esso, gravemente eroso dall'azione evangelizzatrice dei missionari prima, e seriamente minacciato dall'impatto distruttore e omologante del colonialismo imperialista poi, non vada irrimediabilmente perduto.

È così che la lingua, il fabulario mitico, le credenze, i rituali, come pure le strutture economiche e sociali, le tecniche di produzione della vita materiale, la sapienza utile alla sopravvivenza quotidiana, rivestendo agli occhi dello studioso pari rilevanza e dignità, vengono studiati e documentati oggettivamente, senza schemi mentali pregiudiziali e senza atteggiamenti dogmatici. 
Se questo lavoro, di cui si ha ampia prova sfogliando le pagine dei Vocabolari, è di altissimo livello, e se le brillanti analisi del paesaggio e degli insediamenti umani, contenute nei diari di viaggio, evocano un'affascinante figura di geografo umanista, è la ricerca condotta a proposito del Jurupari, che rientra a tutti gli effetti nell'ambito dell'etnografia religiosa, ad apparirci come l'impresa più straordinaria compiuta dall'esploratore.

Come osserva giustamente Padre Alcionilio Brüzzi Alves da Silva (1962, p. 316 e segg.), in un ampio studio dedicato alle civiltà indigene dell'Uaupés, la religione costituisce uno dei capitoli più difficili dell'etnografia di queste tribù, sia per la presenza di un'influenza del cristianesimo che data dall'epoca della scoperta, sia per la forte reticenza e diffidenza dell'indio a trattare di argomenti religiosi con il bianco, reticenza che in certi casi è dovuta ad un vero e proprio divieto di rivelare contenuti ritenuti segreti.

Dopo aver analizzato in dettaglio l'osservanza del culto di Jurupari negli indigeni della missione di Jauareté, presso la quale conduceva la propria ricerca, Brüzzi da Silva conclude che non è vero che gli indios dell'Uaupés non conoscono Dio: non conoscono Gesù Cristo, il Dio dei bianchi, ma conoscono, temono e venerano Jurupari. «Wak-ti (Jurupari) non è semplicemente un Eroe della tribù, è il Legislatore, pertanto il Signore Supremo. Egli punisce o punirà i trasgressori. Per scongiurare i suoi castighi, bisogna ingraziarselo con riti appositi» (ivi, p. 357 e segg).

Stradelli, grazie ad un'intelligenza vivida e ad un'innata capacità di comunione con l'altro e il diverso, non solo aveva intuito, più di mezzo secolo prima, quello che per l'etnologo brasiliano costituisce un punto d'arrivo, ma era riuscito ad avere accesso a contenuti sacri e segreti che solo potevano essere rivelati a «fratelli» (indios appartenenti alla stessa tribù) «adulti» (già iniziati).

Per capire l'eccezionalità di quest'attitudine, bisogna ricordare che l'esploratore era uomo dell'ottocento, formatosi in una temperie culturale dominata dal positivismo e dagli schemi evoluzionistici, nella quale la missione delle nazioni «civilizzate» condotta non più in nome della fede, ma in quello della ragione - era quella di portare il progresso e la civiltà ai popoli «selvaggi».

Nel suo approccio spoglio di preconcetti e nel considerare ogni comportamento nel quadro della cultura in cui esso è radicato ed assume un significato, Stradelli è 
sicuramente un precursore del relativismo culturale. Spesso si trovò a dover constatare, in relazione a fatti economici e sociali, la violenza della cultura straniera dominante su quella indigena, e in questi casi condannò senz'appello la società «civilizzatrice». Abbiamo potuto mostrare come tale violenza si esplicò, nel corso dei secoli, anche nei confronti dei beni immateriali - credenze e culti religiosi, cosmogonia e mitologia, sapere tradizionale - i quali, mantenendo l'identità e l'unità delle comunità indigene, devono esserne considerati il patrimonio più prezioso.

L'opera compiuta dallo studioso a proposito del mito di Jurupari - e di altri temi mitologici indigeni - può essere ancor meglio compresa e valorizzata in questa prospettiva, che lo vede dar voce e scrittura, in un'operazione di delicata mediazione, all'immaginario dei popoli della foresta, assicurandogli una dimora amica, un riparo dalle insidie del tempo, dell'oblio e del «progresso».

In una lettura incredibilmente anticipatrice del lavoro etnografico come traduzione, riuscirà a realizzare una felice trasposizione di codici e significati, costruendo ponti tra una lingua (nhenggatu e dialetti indigeni) e l'altra (italiano o portoghese), tra il racconto orale e la parola scritta, e soprattutto, tra i paradigmi e i valori delle civiltà «senza scrittura» e quelli, universalizzanti, del mondo occidentale (cfr. EVANS-PRITCHARD, 1950, p. 63 e pp. 79-82).

Il personaggio di Stradelli ci appare, significativamente, come opposto e complementare a quello di Jurupari - «colui che sigillò la nostra bocca» - , per essere, per sempre, colui che dà voce ed espressione a ciò che accadde «nel principio del mondo», la cui memoria è legata al filo dorato della conoscenza.

\section{NOTE}

${ }^{1}$ «Pellegrino, venuto da terre straniere, ma di questa terra, più che figlio, amante, alzo la flebile voce, a cui danno forza l'amore e il canto»(STRADELLI, 1900, p. 96, TdA). Tutte le citazioni contenute nel presente testo, provenienti da opere redatte in lingue diverse dall'italiano, sono tradotte dell' autrice.

${ }^{2}$ È da ritenere che Stradelli fu il primo ad utilizzare la macchina fotografica nella foresta amazzonica, servendosene già nelle prime esplorazioni, che risalgono al 1880. È solo nella prima decade del secolo $\mathrm{XX}$, infatti, che la fotografia comincia ad essere utilizzata dagli etnologi in modo sistematico, come 
ausilio alla ricerca sul campo. L'esploratore tedesco Koch Grunberg (1872-1924), che viaggiò in Amazzonia tra il 1903 e il 1905, ne è un esempio.

${ }^{3}$ Mayua, il grande serpente, è per gli indios l'essere misterioso da cui proviene il male, il cui figlio (raira) possiederebbe al contrario attributi supremi di bontà. È l'esploratore Henri Coudreau a riferire di questo soprannome dato a Stradelli dagli indios (cit. in CÂMARA CASCUDO, 1936, p. 82).

${ }^{4}$ Pubblicò nel 1877 Una gita alla Rocca d'Olgisio, un poemetto in versi e, l'anno successivo, Tempo sciupato, una raccolta di poesie in varie metriche.

${ }^{5}$ Lo nheengatu, conosciuto anche come lingua geral o lingua boa, è una lingua della famiglia tupiguarani, parlata dagli indios della costa settentrionale del Brasile, che fu utilizzata, su impulso dei missionari gesuiti e sino alla fine dell'ottocento, come lingua franca nel territorio amazzonico. Stradelli è contrario all'opinione, diffusa al tempo del suo arrivo in Amazzonia, per la quale la lingua boa fosse una creazione artificiale dei Gesuiti. Riconosce, d'altra parte, che questi diffusero l'idioma ben oltre l'ambito originario, insegnandolo anche alle tribù dell'interno.

${ }^{6}$ I seringueiros sono i raccoglitori del lattice. Va ricordato che Stradelli fu testimone dell'intero ciclo economico del caucciù (1890-1910), che vide Manaus trasformarsi rapidamente in una delle città più ricche del mondo e rivaleggiare, per la grandiosità delle strutture e l'intensa vita culturale e mondana, con le maggiori capitali europee.

${ }^{7}$ Questi studiosi, come Stradelli, erano animati dalla volontà di diffondere la conoscenza delle lingue indigene e del folclore amazzonico. Assieme, costituirono una sorta di gruppo di studio, scambiandosi continuamente informazioni e lavorando spesso congiuntamente.

${ }^{8}$ Di tali esplorazioni esistono i dettagliati resoconti pubblicati sul Bollettino della Società Geografica Italiana tra il 1887 e il 1900 : Nell'Alto Orenoco; Dal Cucuhy a Manaos, Rio Branco, L'Uaupés e gli Uaupés, Iscrizione Indigene nella regione dell'Uaupés.

9 I Vocabulários furono pubblicati solo nel 1929, tre anni dopo la morte di Stradelli, sulla Rivista dell'Istituto Storico e Geografico Brasiliano (IHGB).

${ }^{10}$ All'esploratore è dedicato il libro di Danilo Manera Yurupari. I flauti dell'anaconda celeste (Manera, 1999). In appendice al volume è riportato integralmente il testo de La leggenda del Jurupary. Non risultano altre monografie. ${ }^{11}$ Sono di recente pubblicazione, per il Brasile: Lendas e contos de viagem (2009), che reca i resoconti di
viaggio e le leggende mitologiche in traduzione portoghese, a cura di Aurora Foroni Bernardini, preceduti
da un saggio di quest'ultima; Makunaíma e Jurupari. Cosmogonias Amerindias (2002), a cura di Sergio
Medeiros, dove il lavoro di Stradelli sul Jurupari viene trattato parallelamente a quello di Koch Grünberg
su Makunaíma. Va menzionato inoltre il lavoro del colombiano Héctor Orjuela, Yurupary, mito, leyenda
y epopéia del Vaupés (1984), studio approfondito del mito di Jurupari in cui la Leggenda di Stradelli,
allegata al volume nella versione in spagnolo di Susanna Alessi, assume un ruolo centrale ed è analizzata
in dettaglio.

12 La manifestazione «Momento Italia-Brasile» ha avuto l'obiettivo di celebrare l'amicizia ed intensificare le relazioni economiche e culturali tra i due paesi. Le iniziative dedicate a Stradelli si sono tenute nei mesi di maggio e giugno del 2012, presso la sede dell'Università Statale di Rio de Janeiro (UERJ). La mostra fotografica, intitolata $O$ filho da serpente encantada. Fotografias de Ermanno Stradelli, è stata realizzata in collaborazione con la Società Geografica Italiana.

13 Stradelli pubblicò, per l'editore piacentino Vincenzo Porta, due volumetti di leggende mitologiche amerindie : Eiara: leggenda tupi-guarani (1885); Duas lendas amazônicas, in cui sono narrate, in portoghese, due leggende di argomento storico, Pitiapo e Ajuricaba, che si ispirano a vicende datate tra

Geo UERJ - Ano 14, nº. 23, v. 2, $2^{\circ}$ semestre de 2012 p. 331-361

ISSN: 1415-7543 E-ISSN: 1981-9021

http://www.e-publicacoes.uerj.br/index.php/geouerj 
la fine del XVII e l'inizio del XVIII secolo, di cui sono protagonisti gli indios Tariana e Manaos. Pubblicò inoltre, con lo stesso editore, La confederazione dei Tamoi. Poema epico, traduzione italiana dell'opera indianista A confederação dos Tamoyos, di Domingo José Gonçalves de Magalhães.

14 «Cinquant'anni di catechesi furono sufficienti ad attribuire a Jurupari una reputazione satanica [...] Già nel secolo XVII il Figlio del Sole, il Signore degli Strumenti musicali, il Custode dei Segreti evocato al suono delle maracas rituali era, definitivamente e dalla testa ai piedi, il Diavolo, Belzebù, Satanasso» (CÂMARA CASCUDO, 1947 p. 68).

${ }^{15}$ Così il francese Jean de Lery, alla metà del '500: «Bisogna considerare che questa povera gente è in vita continuamente afflitta da questo spirito maligno (Aygnan). [...] Alcuni anche dicevano di vederlo materialmente, ora sotto forma di bestia o d'uccello, ora sotto altre forme strane. E si meravigliavano assai nell'accorgersi che noi non ne venivamo assaliti mai. Noi rispondevamo allora che tale incolumità veniva da Dio, del quale parlavamo loro spesso... A volte, sentendosi oppressi, promettevan di credere come noi». (Cit. in SEPPILLI, 1964, p. 437).

16 I pajés, depositari dei segreti di quella devozione ed unico tramite tra gli indigeni e la divinità, costituivano, per l'influenza che esercitavano nella vita delle tribù, un ostacolo all'azione evangelizzatrice e il principale nemico nella lotta per la salvezza delle anime. I gesuiti erano convinti che i poteri dei pajés e il loro sapere medico, metereologico e topografico provenissero dal demonio (cfr. CÂMARA CASCUDO, cit., p. 87) .

${ }^{17}$ Couto de Magalhães è considerato da Câmara Cascudo l'iniziatore degli studi del folclore in Brasile. Lo studioso accoglie l'interpretazione secondo la quale la parola Jurupari significherebbe «essere che viene al nostro giaciglio» (MAGALHÃES, 1876, p. 83).

${ }^{18}$ I missionari francescani raggiunsero la regione dell'Uaupés nel 1879. Frate Venanzio Zilochi aveva fondato la missione di Taraquá, nei pressi della foce del fiume Tiquié. Padre Coppi, arrivato a Manaus nel 1882, fondò, insieme a Padre Canioni, le missioni di Jauareté e Ipanoré, nell'alto Uaupés.

19 Il frate vendette «l'infame simulacro» nel 1884 al Museo Kircheriano - oggi Museo Preistorico Etnografico Luigi Pigorini - insieme ad altri oggetti rituali.

${ }^{20}$ Secondo questa versione, Jurupari è un essere malvagio e vendicativo, che mangia i bambini e violenta le donne. Per questo viene ucciso dagli uomini in un rogo e dalle sue ceneri nascono le palme pasciuba, dalle quali sono ricavati gli strumenti sacri (COUDREAU, 1887, p. 184 e segg.).

${ }^{21}$ Un'etimologia che può esprimere lo stesso concetto è quella suggerita da Padre Costantino Tastevin, per il quale iu-ru-pari vorrebbe significare «mettere una maschera al proprio volto o bocca», da pari, maschera e rua, bocca o volto (cit. in CÂMARA CASCUDO, 1958, p. 101).

${ }^{22}$ Maximiano collaborò con Antônio Brandão de Amorim alla stesura dei testi in nheengatu di alcune leggende che avevano raccolto assieme, e che verranno pubblicate da quest'ultimo sulla Revista do Instituto Histórico e Geográfico Brasileiro (AMORIM, 1926).

${ }^{23}$ È a Maximiano José Roberto che Luís da Câmara Cascudo dedica il volume Em Memória de Stradelli.

${ }^{24}$ Quello raccolto da Maximiano è il ciclo tariana del mito di Jurupari, che si ritrova, nelle comunità indigene dell' Amazzonia nord-occidentale, in differenti versioni. Per una rassegna dei principali cicli, si veda Orjuela, op.cit., p. 70 e segg.

25 «In principio volevo farne un riassunto, ma poi mutai pensiero, e lo tradussi; ed è questa traduzione che aggiungo, in seguito a queste note, senza maggiori commenti» (STRADELLI, 1890a, p. 452).

Geo UERJ - Ano 14, nº 23, v. 2, $2^{\circ}$ semestre de 2012 p. 331-361

ISSN: 1415-7543 E-ISSN: 1981-9021

http://www.e-publicacoes.uerj.br/index.php/geouerj 
${ }^{26}$ La Leggenda, che ha un'estensione di circa 60 pagine, fu pubblicata in due parti, su fascicoli distinti del Bollettino: la prima parte nel fascicolo VII - VIII, la seconda nel fascicolo IX, della serie III - volume III del 1890 (rispettivamente pp. 660-689 e 798-835). Nel 1964 il testo fu ripubblicato in Brasile, a São Paulo, in un volumetto a cura dell'Istituto Culturale Italo-Brasiliano (ICIB), dal titolo La leggenda del Jurupary e outras lendas amazônicas, che include anche le leggende Pitiápo e la Leggenda del Taria.

${ }^{27}$ La leggenda di Barbosa Rodrigues, fu pubblicata nel 1890, con il titolo di A lenda sagrada de Izy o Bokan, in Poranduba Amazonense. Izy è il nome di Jurupari in lingua tariana.

${ }^{28}$ Come sottolinea Gordon Brotherston, il testo contiene parole in lingua tucana, aruaca, baniwa, cubeua ed altri idiomi parlati in una regione caratterizzata da un' ampia varietà linguistica. «Come per confermare quest'assetto di federalismo linguistico, Jurupari sceglie termini di più di dieci dialetti locali per battezzare i diversi strumenti del suo culto» (2002, cit., p. 398)

${ }^{29}$ L'antropologo paraguayo León Cadogan ha raccolto la tradizione mitologica dei Guarani della Guaira in Ayvu-Rpyta (1959). Il tedesco Kurt Nimuendaju registrò per primo la cosmologia guarani in Lendas da criação e destruição do mundo como fundamentos da religião dos Apapocuva-guarani (1917).

\section{RIFERIMENTI BIBLIOGRAFICI}

AMORIM, A. Brandão de, Lendas em Nheengatu e em Português, «Revista do Instituto Histórico Geográfico Brasileiro», Rio de Janeiro, vol. 154, 1926, p. 9-479.

BARBOSA RODRIGUES, João, Poranduba Amazonense, «Anais da Biblioteca Nacional», Rio de Janeiro, t. XIV, 1890.

BETTENCOURT, Gastão de, A Amazônia no fabulário e na arte, Lisboa: Pro Domo, 1946.

BIOCCA, Ettore, Viaggio tra gli Indi. Appunti di un biologo, Roma: C.N.R., 1965.

BRUZZI ALVES DA SILVA, Alcionilio, A civilização indígena no Uaupés, São Paulo: Centro de Pesquisas de Iauareté, 1962.

BROTHERSTON, Gordon, Sá, Lúcia, Peixes, constelações e Jurupari: a pequena enciclopédia amazônica de Stradelli, São Paulo, «Revista do Museu de Arquelogia e Etnologia», n. 14, 2004, pp. 345-358.

Geo UERJ - Ano 14, nº. 23, v. 2, $2^{\circ}$ semestre de 2012 p. 331-361

ISSN: 1415-7543 E-ISSN: 1981-9021

http://www.e-publicacoes.uerj.br/index.php/geouerj 
BROTHERSTON, Gordon, Jurupari articula o espaço dos Tária e a ciência da América Tropical, in MEDEIROS (org.), Makunaíma e Jurupari. Cosmogonias Amerindias, São Paulo : Perspectiva, 2002, pp. 397-413.

CÂMARA CASCUDO, Luís, Em memória de Stradelli, Manaus: Livraria Clássica, 1936.

CÂMARA CASCUDO, Luís, Geografia dos mitos brasileiros, Rio de Janeiro: Livraria José Olímpio Editora, 1958.

CÂMARA CASCUDO, Luís, Dicionário do Folclore Brasileiro, Rio de Janeiro, 1954 [2 ed. Instituto Nacional do Livro, 1959].

COLINI, Giuseppe Angelo, La Provincia delle Amazzoni, secondo la Relazione del P. Giuseppe Illuminato Coppi Missionario nel Brasile, «Bollettino della Società Geografica Italiana», Roma, serie II - vol X, pp. 136-141 e 193-204, 1885.

COPPI, Giuseppe Illuminato, Appunti interessanti del Missionaria Apostolico francescano nell'America del Sud, Siena: Tip. Arciv. S. Bernardino, 1897.

COUDREAU, Henri Anatole, La France équinoxiale, 2 voll., Paris: Challamel Ainé, 1887.

CURATOLA, Marco, Makakaráua. La sacra maschera di Jurupari, in Indios del Brasile. Culture che scompaiono, catalogo della mostra a cura di Fausto Zevi, Roma: De Luca Editore, 1983.

DUNDES, Alan, Structural Typology in North American Indian Folk-tales, New Jersey: Prentice Hall, 1965.

EVANS-PRITCHARD, E.E., Social Antropology, London: Routledge \& Kegan Paul, 1972 [tr. por. Antropologia Social, Lisboa: Edições 70, 2011].

FAULHABER, Priscila, Nos varadouros das representações: redes etnográficas na Amazônia do início do século XX, «Revista de Antropologia», São Paulo, vol 40/2, pp. 101-143, 1997.

HUMBOLDT, Alexander von, Voyage aux Régions Equinoxiales du Nouveau Continent, 3 voll., Paris, 1814-1825 [tr. it. Viaggio alle Regioni Equinoziali del Nuovo Continente, 3 voll., Fratelli Palombi, Roma, 1986].

Geo UERJ - Ano 14, nº. 23, v. 2, $2^{\circ}$ semestre de 2012 p. 331-361 ISSN: 1415-7543 E-ISSN: 1981-9021 http://www.e-publicacoes.uerj.br/index.php/geouerj 
ISENBURG, Teresa (a cura di), Naturalistas Italianos no Brasil, São Paulo: Icone Editora, 1991.

LÉVI-STRAUSS, Claude, Anthropologie structurale, Paris : Plon, 1958 [tr. port. Antropologia Estructural, São Paulo: Cosac Naify, 2008].

LÉVI-STRAUSS, Claude, Du miel aux cendres (Mythologiques II), Paris: Plon, 1973 [tr. port. Do mel às cinzas - Mitologicas v.2, São Paulo: Cosac Naify, 2004].

LÉVI-STRAUSS, Claude, Myth and Meaning, University of Toronto Press, Toronto, 1978 [tr. port. Mito e significado, Lisboa: Edições 70, 2010].

MAGALHÃES, Couto de, O Selvagem, Rio de Janeiro: Biblioteca Nacional, 1876.

MALINOWSKI, Bronislaw, Argonauts of the Western Pacific, London: Routlege \& Sons, 1922. Disponibile in Internet: http://archive.org/details/argonautsofthewe032976mbp

MANERA, Danilo, Yurupari. I flauti dell'anaconda celeste, Milano: Feltrinelli, 1999.

MAURO, Alessandra, Il mito e la rima: la trasposizione poetica delle leggende amazzoniche raccolte da Ermanno Stradelli, «Letterature d'America», Roma, n. 19/20, pp. 113-128,1983.

MEDEIROS (Org.), Sergio, Makunaíma e Jurupari. Cosmogonias Amerindias, São Paulo : Perspectiva, 2002.

MINDLIN, Betty, e narradores SURUI PATER, Vozes da Origem, Rio de Janeiro: Record, 2007.

ORICO, Osvaldo, Mitos ameríndios e crendices amazônicas, Rio de Janeiro: Civilização brasileira, 1975.

ORJUELA, Héctor H., Yurupary. Mito, leyenda y epopeya del Uaupés, Bogotà: Instituto Caro y Cuervo, 1983.

PELOSO, Silvano, Medioevo nel sertão, Napoli: Liguori, 1984.

PELOSO, Silvano, Amazzonia. Mito e letteratura del mondo perduto, Roma: Editori Riuniti, 1988. 
SÁ, Lúcia, A lenda de Jurupari : texto sagrado o fruto da imaginação de littérateurs? , in MEDEIROS (org.), Makunaíma e Jurupari. Cosmogonias Amerindias, São Paulo : Perspectiva, 2002, pp. 347-358.

SEPPILLI, Anita e Tullio, L'esplorazione dell'Amazzonia, Torino: Utet, 1964.

STRADELLI, Ermanno, Eiara: leggenda Tupi-Guarani, Piacenza: Porta, 1885.

STRADELLI, Ermanno, La confederazione dei Tamoi. Poema epico di D. J. Magalhães, Piacenza: Porta, 1885.

STRADELLI, Ermanno, Nell'alto Orenoco, Roma: Società Geografica Italiana Editore, 1888.

STRADELLI, Ermanno, Dal Cucuhy a Manaos, Roma: Società Geografica Italiana Editore, 1889.

STRADELLI, Ermanno, Rio Branco, Roma: Società Geografica Italiana Editore, 1889.

STRADELLI, Ermanno, L'Uaupés e gli Uaupés, «Bollettino della Società Geografica Italiana», Roma, serie III - vol III, pp. 425-453, 1890.

STRADELLI, Ermanno, La Leggenda del Jurupary, «Bollettino della Società Geografica Italiana», Roma, serie III - vol III, pp. 660-689 e 798-835, 1890.

STRADELLI, Ermanno, Leggende dei Taria, «Memorie della Società Geografica Italiana», Roma, vol IV, pp. 141-148, 1896.

STRADELLI, Ermanno, Iscrizioni indigene della regione dell'Uaupés, «Bollettino della Società Geografica Italiana», Roma, serie IV - vol I, pp. 457- 483, 1900.

STRADELLI, Ermanno, Duas lendas amazônicas, Piacenza: Porta, 1900.

STRADELLI, Ermanno, Vocabulários da língua geral portuguez-nheengatu e nheengatuportuguez, precedidos de um esboço de grammatica nheengatu-umbue-saua miri e seguidos de contos em língua geral, «Revista do Instituto Histórico Geográfico Brasileiro», Rio de Janeiro, t. 104 , vol 158, pp. 5-678, 1929.

STRADELLI, Ermanno, La leggenda del Jurupary e outras lendas amazônicas, São Paulo, Instituto Cultural Italo-Brasileiro, 1964, Caderno IV. 
STRADELLI, Ermanno, Lendas e notas de viagem. A Amazônia de Ermanno Stradelli, São Paulo: Martins Editora, 2009.

WALLACE, Alfred Russel, A Narrative of travels on the Amazon and Rio Negro, with an account of the native tribes, and observations on the climate geology, and natural history of the Amazon Valley, London, 1853 [2 ed. Yale University Press, New Haven, 1969].

Artigo encaminhado para publicação em novembro de 2012.

Artigo aceito para publicação em novembro de 2012.

Geo UERJ - Ano 14, nº. 23, v. 2, $2^{\circ}$ semestre de 2012 p. 331-361 ISSN: 1415-7543 E-ISSN: 1981-9021

http://www.e-publicacoes.uerj.br/index.php/geouerj 